\title{
Empirically derived dietary patterns and incident type 2 diabetes mellitus: a systematic review and meta-analysis on prospective observational studies
}

\author{
Zahra Maghsoudi ${ }^{1,2}$, Reza Ghiasvand ${ }^{1,2}$ and Amin Salehi-Abargouei ${ }^{3,4, *}$ \\ ${ }^{1}$ Food Security Research Center, Isfahan University of Medical Sciences, Isfahan, Islamic Republic of Iran: \\ ${ }^{2}$ Department of Community Nutrition, School of Nutrition and Food Science, Isfahan University of Medical Sciences, \\ Isfahan, Islamic Republic of Iran: ${ }^{3}$ Nutrition and Food Security Research Center, Shahid Sadoughi University of \\ Medical Sciences, Yazd, Islamic Republic of Iran: ${ }^{4}$ Department of Nutrition, Faculty of Health, Shahid Sadoughi \\ University of Medical Sciences, PO Box 887-89165, Yazd, Islamic Republic of Iran
}

Submitted 25 May 2014: Final revision received 24 February 2015: Accepted 25 February 2015: First published online 28 April 2015

\begin{abstract}
Objective: To systematically review prospective cohort studies about the association between dietary patterns and type 2 diabetes mellitus (T2DM) incidence, and to quantify the effects using a meta-analysis.

Design: Databases such as PubMed, ISI Web of Science, SCOPUS and Google Scholar were searched up to 15 January 2015. Cohort studies which tried to examine the association between empirically derived dietary patterns and incident T2DM were selected. The relative risks (RR) and their $95 \%$ confidence intervals for diabetes among participants with highest $v$. lowest adherence to derived dietary patterns were incorporated into meta-analysis using random-effects models.

Results: Ten studies ( $n$ 404 528) were enrolled in the systematic review and metaanalysis; our analysis revealed that adherence to the 'healthy' dietary patterns significantly reduced the risk of T2DM $(R R=0.86 ; 95 \%$ CI $0.82,0.90)$, while the 'unhealthy' dietary patterns adversely affected diabetes risk $(\mathrm{RR}=1 \cdot 30$; $95 \% \mathrm{CI}$ $1 \cdot 18,1 \cdot 43)$. Subgroup analysis showed that unhealthy dietary patterns in which foods with high phytochemical content were also loaded did not significantly increase $\mathrm{T} 2 \mathrm{DM}$ risk $(\mathrm{RR}=1 \cdot 06 ; 95 \% \mathrm{CI} 0 \cdot 87,1 \cdot 30)$.

Conclusions: 'Healthy' dietary patterns containing vegetables, fruits and whole grains can lower diabetes risk by $14 \%$. Consuming higher amounts of red and processed meats, high-fat dairy and refined grains in the context of 'unhealthy' dietary patterns will increase diabetes risk by $30 \%$; while including foods with high phytochemical content in these patterns can modify this effect.
\end{abstract}

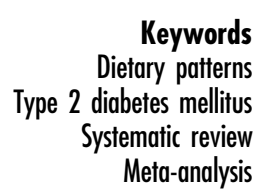

Type 2 diabetes mellitus (T2DM), a chronic lifethreatening metabolic disorder ${ }^{(1)}$, is spreading globally at an alarming rate ${ }^{(2)}$. For instance, reports show that its prevalence has doubled during the last 20 years in the $\mathrm{USA}^{(3)}$ and it is predicted that the number of diabetic people will reach 380 million in 2025 , worldwide ${ }^{(4)}$. While the wide range of accompanying disabilities and lifeaffecting complications can decrease life expectancy by nearly 10 years, the disease and its concerning outcomes are preventable by controlling its contributing factors ${ }^{(5)}$.

Several factors including weight gain, physical activity, smoking and also dietary behaviours like fruit and vegetable consumption have been related to T2DM risk in previous studies ${ }^{(6)}$. Dietary intakes of individual nutrients and food groups have long been studied in relation to diabetes ${ }^{(7-9)}$; however, it is proposed that foods may affect chronic disease risk synergistically rather than individually $^{(10)}$. Dietary pattern analysis has emerged recently to combine multiple foods or food groups as a single exposure by using scores assigned to foods or food groups (a priori defined dietary patterns) or statistical approaches (a posteriori defined or empirically derived dietary patterns) ${ }^{(11)}$. Empirical methods use different statistical methods like factor analysis, reduced rank regression or cluster analysis to derive dietary patterns ${ }^{(11)}$. Using a posteriori methods helps researchers to combine 
numerous foods or food groups to derive dietary patterns as single exposure variables ${ }^{(12)}$. It has been assumed that dietary patterns can provide a better and more general insight into diet-disease relationships ${ }^{(12)}$ and may be more predictive of chronic disease risk than individual nutrients or foods because the effects from single nutrients or foods are too small to be detectable ${ }^{(11)}$.

Several studies have tried to assess the effects of dietary patterns in relation to diabetes risk and their results have been contradictory. Some studies have shown a significant association between dietary patterns high in fruits and vegetables and incident $\mathrm{T}_{2} \mathrm{DM}^{(13)}$, while others could not show the same results ${ }^{(14)}$. Studies have also reached conflicting results about the effect of unhealthy dietary patterns highly loaded by red meat and high-fat foods in association with $\mathrm{T}_{2} \mathrm{DM}^{(15,16)}$.

Cohort studies are the best epidemiological approach to reveal the casual role of diet in disease development. Therefore, in the present study, we tried to summarize prospective data about the effect of empirically derived dietary patterns in association with diabetes risk and if possible to quantify the association between the same dietary food patterns and T2DM risk and also search for the sources of the difference between studies' results, using meta-analysis.

\section{Materials and methods}

\section{Search strategy}

Our search was focused on the keywords selected from Medical Subject Headings (MeSH) database and non$\mathrm{MeSH}$ terms related to the topic, including: 'diabetes mellitus', 'diabetes mellitus type 2', 'diabetes', 'dietary pattern', 'food pattern', 'eating pattern', 'dietary habit', 'food habit', 'eating habit', 'dietary behaviour', 'food behaviour', 'eating behaviour', 'diet habit', 'feeding behaviour' and 'feeding pattern'. We searched several databases including PubMed, ISI Web of Science and SCOPUS, up to 15 January 2015. Assurance of finding the maximum related publications was achieved by searching the mentioned keywords in Google Scholar. All of the databases were searched with no language or date limitation. The relevant studies were detected with a hierarchical approach on the basis of titles, abstracts and full text of articles in the first step. In the next step, the full text of all related articles was inspected by reviewers to check the eligibility of the selected papers. Furthermore, reference lists of related articles were checked for any other related paper. All of the steps were performed by two authors (Z.A. and A.S.-A.) separately and any disagreements were resolved through discussion with R.G. as the third author.

\section{Inclusion criteria}

Studies meeting the following criteria were included in our systematic review and meta-analysis: (i) original prospective cohort study; (ii) performed on an adult population; and (iii) as the majority of studies examining the association between dietary patterns and T2DM used exploratory factor analysis or principal component analysis to derive dietary patterns, we included the studies which used the mentioned statistical methods to define the major dietary food patterns.

\section{Exclusion criteria}

Eligible surveys were reviewed carefully by Z.A. for any methodological difference. Studies with the following characteristics were excluded: (i) studies that used an a priori method or statistical methods other than factor analysis to derive dietary food patterns; and (ii) studies that selected different outcomes other than T2DM like insulin resistance, homeostasis model of assessment (HOMA).

\section{Study quality assessment}

The quality of relevant articles was determined using the Newcastle-Ottawa quality assessment scale for cohort studies. An eight-question checklist was filled in for eligible studies ${ }^{(17)}$.

\section{Data extraction}

The following data were extracted from related papers: (i) first author's family name; (ii) publication year; (iii) country in which the study was performed; (iv) participants' sex; (v) participants' age; (vi) statistical analysis method; (vii) duration of follow-up; (viii) study sample size at baseline; (ix) number of cases found after followup; (x) food items that highly loaded on each dietary pattern; and (xi) confounding variables adjusted for in the multivariate model.

Studies conducted on the association between dietary patterns and T2DM found several dietary patterns and named these dietary patterns based on the foods or food groups loaded on each pattern. In the current study we also divided the derived dietary patterns based on the foods loaded on each pattern. If food groups known as healthy - like vegetables, fruits, whole grains and seeds loaded highly on one of these dietary patterns, we named it as a 'healthy' dietary pattern. In contrast, if unhealthy foods - like red meat, processed foods, high-fat dairy and refined grains - loaded highly, we named it as an 'unhealthy' dietary pattern.

The reported relative risks (RR) for T2DM in participants who had the highest adherence to the derived dietary patterns in comparison to those who had the lowest adherence, and their $95 \%$ confidence intervals, were extracted to compute effect sizes. It should be mentioned that some studies used several models to adjust for confounding variables; therefore, we opted for the models with the maximum number of variables. In addition, if multiple publications were found from the same cohort study, data from the most recent report and with the 
longer follow-up period were included. The information was extracted independently by two reviewers (Z.A. and A.S.-A.) and discrepancies were resolved by discussion with the third author (R.G.).

\section{Statistical analysis}

The relative risks and their $95 \%$ confidence limits were extracted to compute $\log (\mathrm{RR})$ values and their corresponding standard errors, which were used as effect size in the current meta-analysis. In addition, to incorporate between-study variation, a random-effects model was used to combine effect sizes. This model takes betweenstudy heterogeneity into account ${ }^{(18)}$. Statistical heterogeneity among studies was evaluated using Cochran's $Q$ test and the $I^{2}$ statistic $^{(19)}$. The sources of heterogeneity were checked by several subgroup analyses ${ }^{(19)}$ based on foods or food groups loaded on dietary patterns, sex, study region, studies' overall quality score, follow-up time, person-years, adjustment for physical activity, adjustment for family history of T2DM, ethnic differences and adjustment for education. To explore the extent of dependency of analyses on a specific study or a particular group of studies, sensitivity analysis was done. Publication bias was evaluated by visual inspection of Begg's funnel plots ${ }^{(20)}$ and the statistical asymmetry of the funnel plot was assessed using Egger's regression asymmetry test and Begg's adjusted rank correlation test ${ }^{(18)}$. Statistical analyses were performed by using the STATA statistical software package version 11.2. $P$ values lower than 0.05 were considered statistically significant.

\section{Results}

The study selection process is described in Fig. 1. Our search retrieved 50920 articles. After scanning titles and abstracts, ten articles were eligible to be included in our systematic review and meta-analysis ${ }^{(13-16,21-26)}$; all of the eligible studies reported a relative risk or hazard ratio for the association between a dietary pattern and risk of T2DM. Four studies were conducted in the USA ${ }^{(13,16,25)}$, two in Europe ${ }^{(21,22)}$, one in $\operatorname{Australia}^{(14)}$ and three in Asia (two in Japan $^{(15,23)}$ and one in China ${ }^{(26)}$ ). A total of 404528 individuals free of T2DM were included in the selected studies at baseline and 18584 cases were diagnosed during the follow-up period. Participants' age ranged between 27 and 84 years. Diabetes incidence was defined according to the WHO classification, as elevated random or fasting plasma glucose level, or elevated plasma glucose $2 \mathrm{~h}$ after a glucose load. All studies used a validated FFQ with sixteen to 178 food items to assess dietary intake. Studies adjusted the estimated risk for confounding variables such as age, sex, smoking, physical activity, energy intake, BMI and history of diseases. Table 1 shows detailed information about the studies included in the systematic review and meta-analysis.

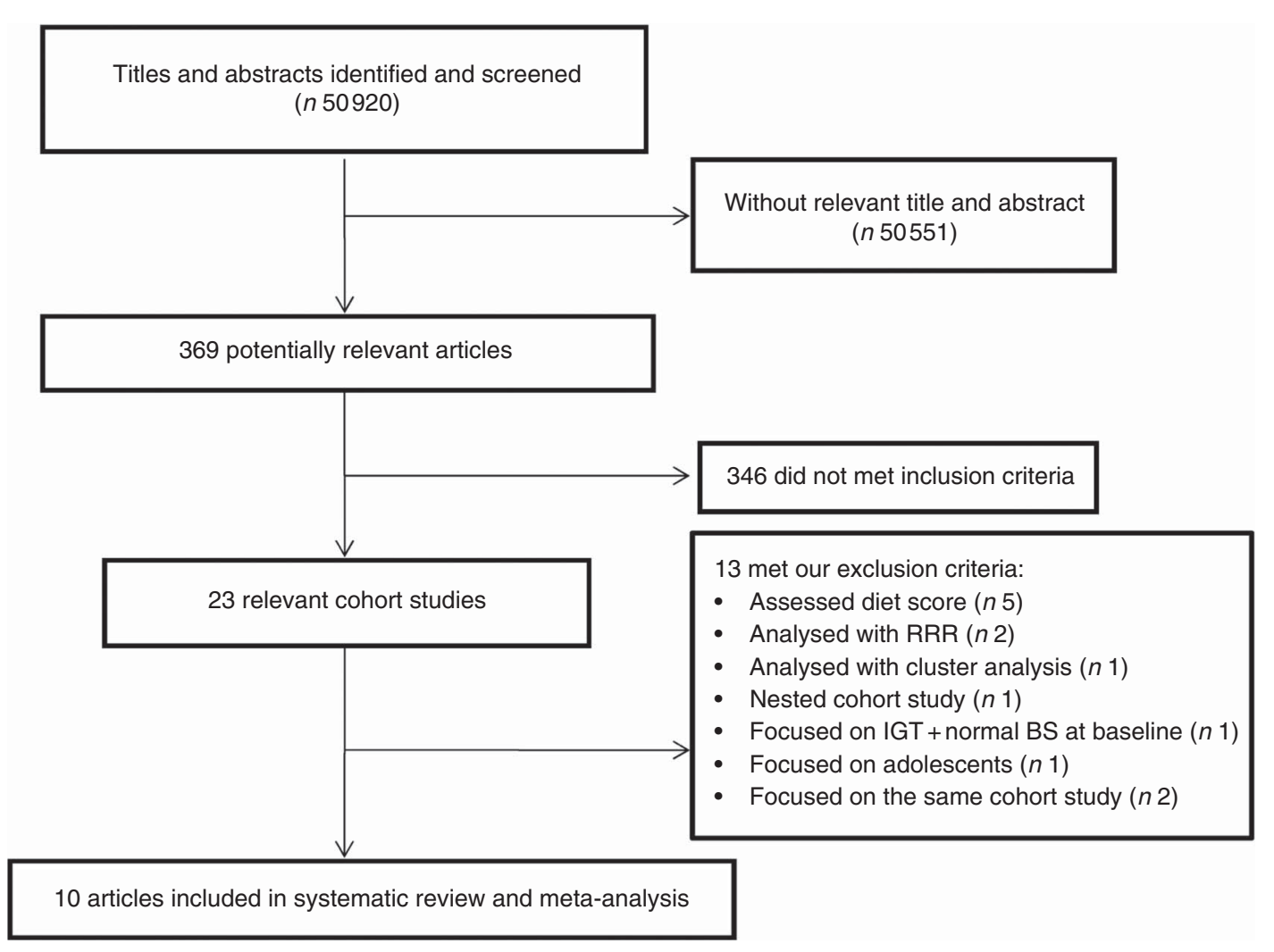

Fig. 1 Flowchart of the study selection process (RRR, reduced rank regression; IGT, impaired glucose tolerance; BS, blood sugar) 


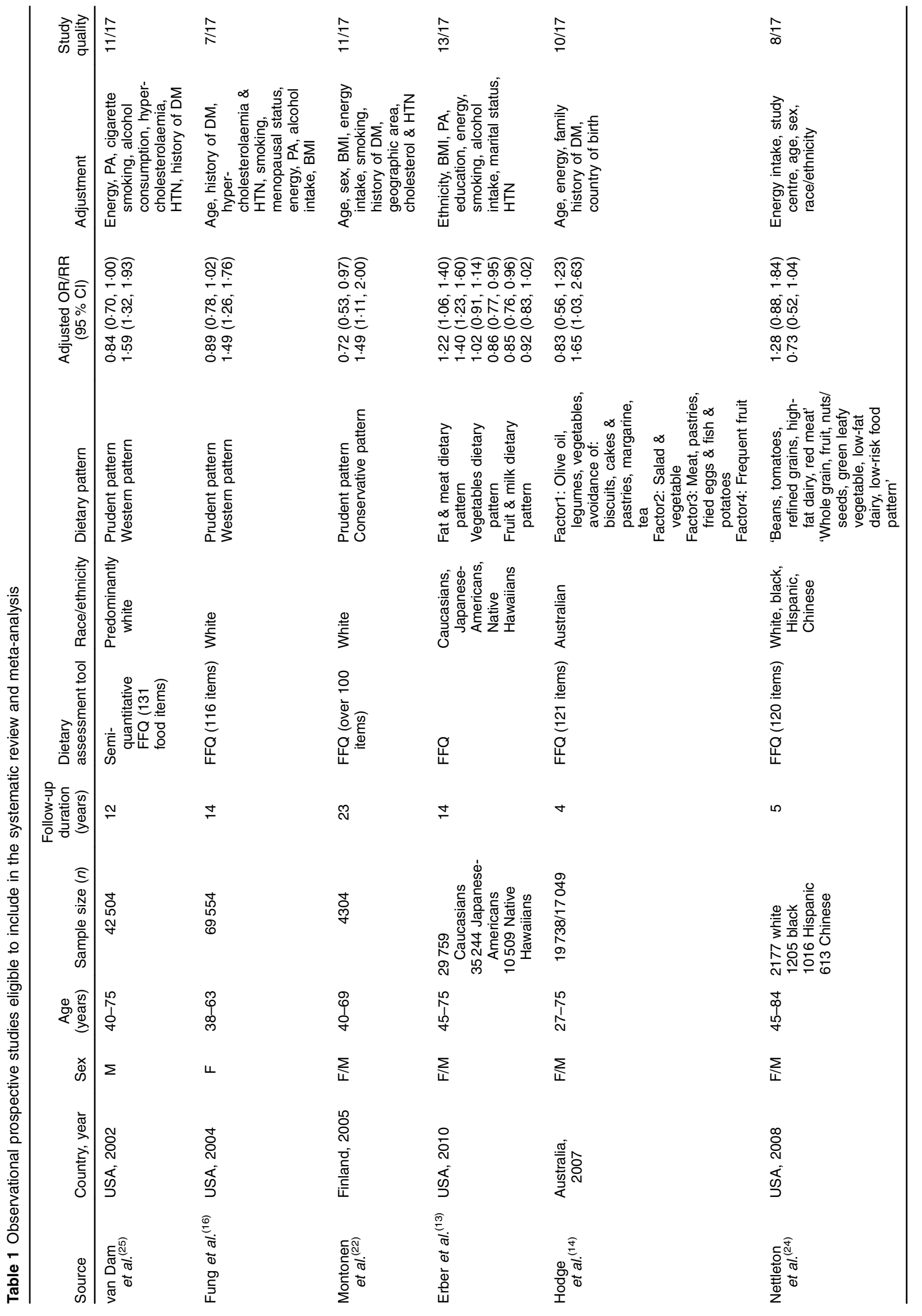




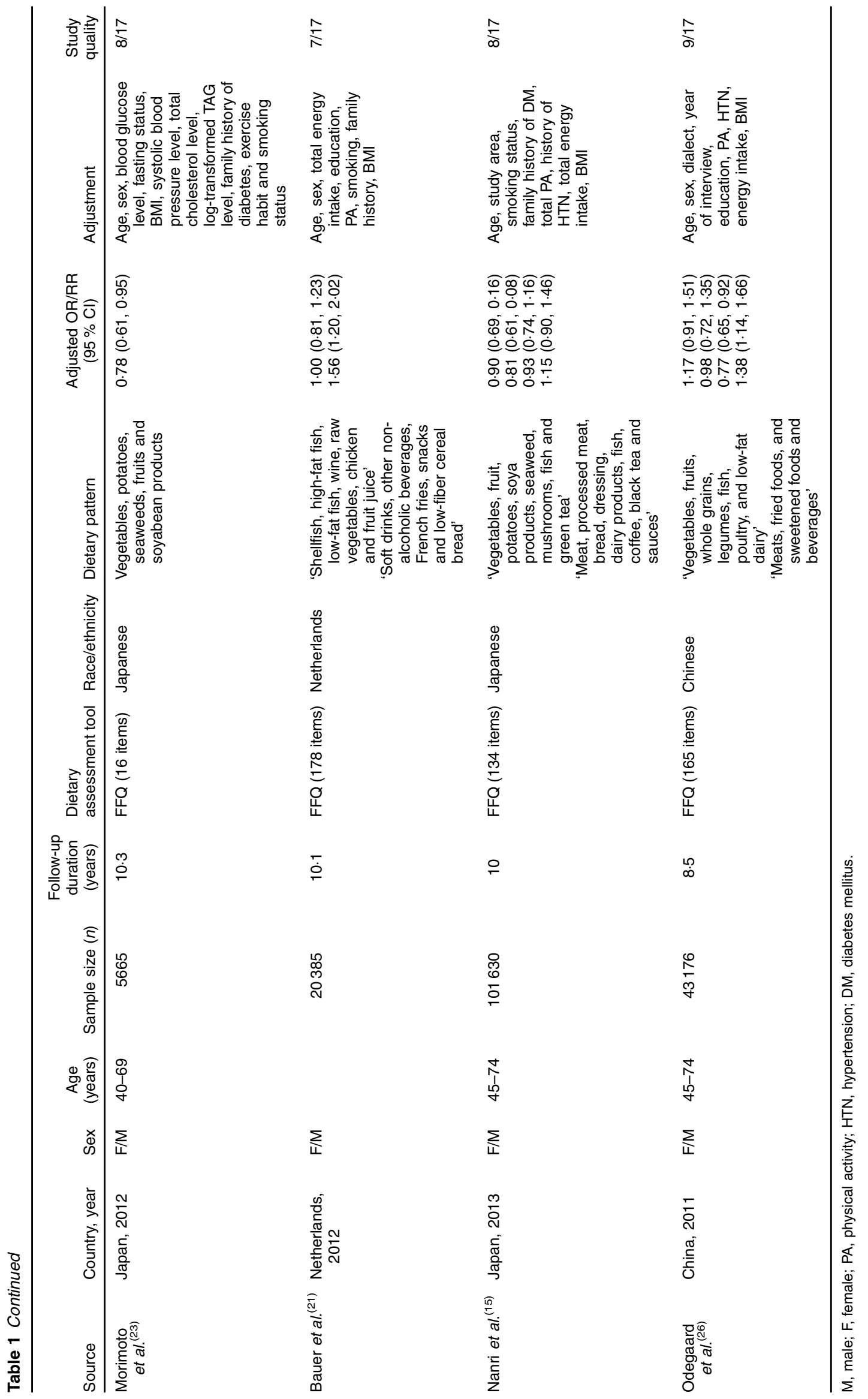


Dietary patterns and type 2 diabetes

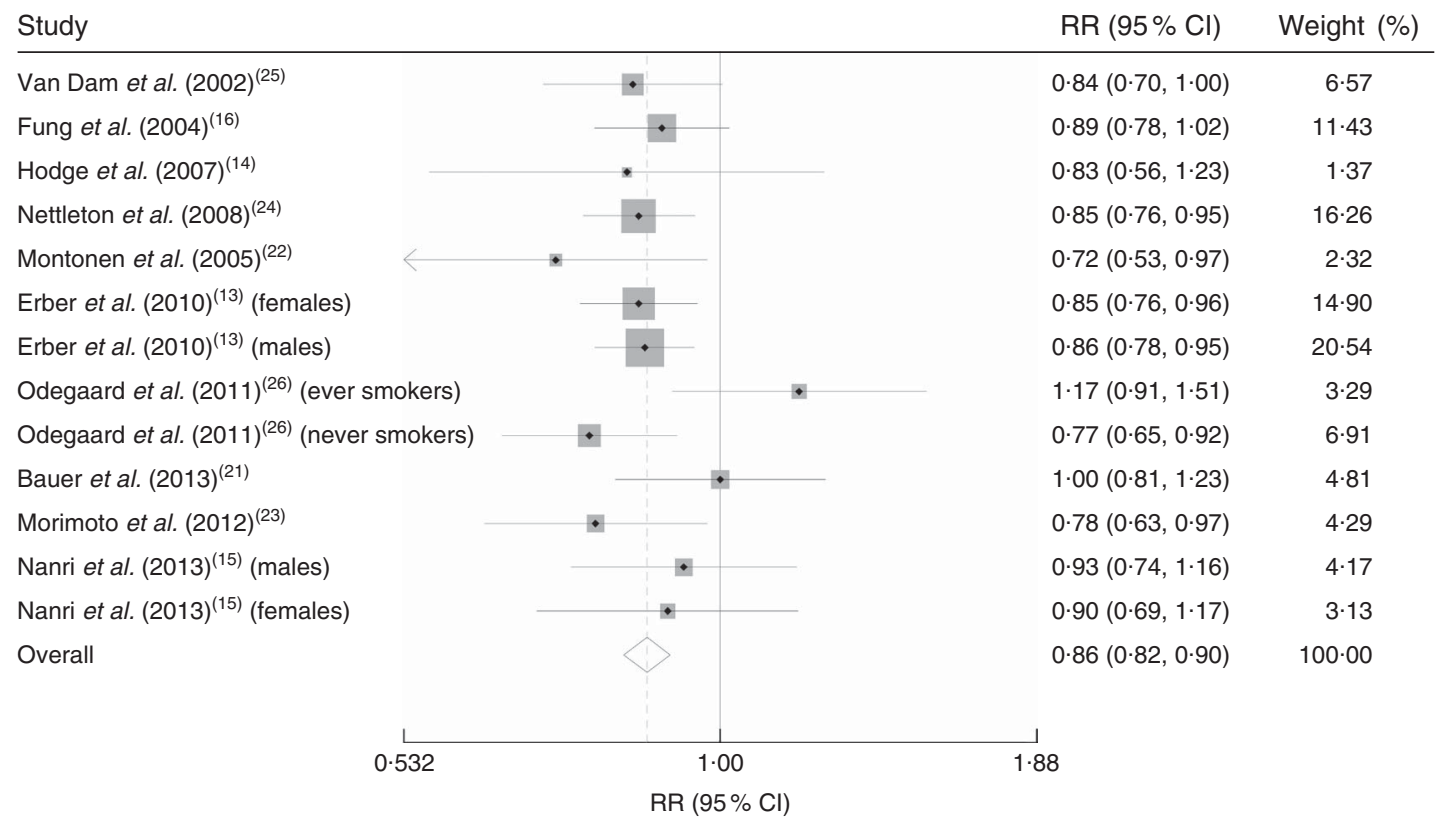

Fig. 2 Forest plot illustrating weighted relative risk (RR) using a random-effects model for the comparison of incident type 2 diabetes mellitus in participants with highest $v$. lowest adherence to 'healthy' dietary patterns. The study-specific RR and $95 \% \mathrm{Cl}$ are represented by the grey square and horizontal line, respectively; the area of the grey square is proportional to the specific-study weight to the overall meta-analysis. The centre of the diamond presents the pooled RR risk and its width represents the pooled $95 \% \mathrm{Cl}$

Two major dietary patterns were derived in almost all included studies, based on the foods and food groups highly loaded on each pattern. Food groups like vegetables, fruits, whole grains and seeds loaded highly on one of these dietary patterns which we called 'healthy' dietary patterns, while in another group of patterns unhealthy foods like red meat, processed foods, high-fat dairy and refined grains loaded highly; therefore we called them 'unhealthy' dietary patterns (Table 1). Our analysis revealed that adhering to the 'healthy' dietary patterns led to a decreased risk of T2DM (RR $=0 \cdot 86$; $95 \%$ CI 0.82, 0.90; Fig. 2); while adhering to the 'unhealthy' dietary patterns increased T2DM risk ( $R R=1 \cdot 30 ; 95 \%$ CI 1·18, 1.43; Fig. 3). There was no evidence of significant between-study heterogeneity for 'healthy' dietary patterns (Cochrane $Q$ test, $P=0.423 ; I^{2}=2.3 \%$ ); however, we observed statistically significant heterogeneity for 'unhealthy' dietary patterns (Cochrane $Q$ test, $P=0.001 ; I^{2}=64.1 \%$ ). Therefore we performed a subgroup analysis based on the food groups loading on 'unhealthy' dietary patterns derived in each study. Beans and tomatoes loaded highly on the derived 'unhealthy' dietary pattern in the study done by Nettelton et $a l^{(24)}$, and food items like coffee, black tea and oolong tea also loaded highly on the 'unhealthy' dietary pattern in the study accomplished by Nanri et al. ${ }^{(15)}$. Although these food groups loaded highly on 'unhealthy' dietary patterns, they are known as sources of bioactive phytochemical compounds; therefore we categorized studies based on whether foods with high phytochemical content loaded on 'unhealthy' dietary patterns or not (Fig. 4). Our subgroup analysis revealed that high consumption of 'unhealthy' foods without consumption of phytochemical-rich foods significantly increased the risk of T2DM $(R R=1.39 ; 95 \%$ CI $1.28,1.52)$, the between-study heterogeneity was not statistically significant in this subgroup (Cochrane $Q$ test, $P=0.142, I^{2}=34.5 \%$ ); while consumption of foods with high photochemical content along with 'unhealthy' foods did not increase the disease risk $(\mathrm{RR}=1 \cdot 06 ; 95 \%$ CI $0 \cdot 87$, $1 \cdot 30$ ), heterogeneity was not statistically significant in this subgroup too (Cochrane $Q$ test, $P=0.06 ; I^{2}=65.7 \%$ ).

We also performed several subgroup analyses based on sex, study region, studies' overall quality score, follow-up time, person-years, adjustment for physical activity, adjustment for family history of T2DM, ethnic differences and adjustment for education for the effect of 'healthy' and 'unhealthy' dietary patterns on T2DM risk. Our analyses showed that 'healthy' dietary patterns reduced the risk of diabetes in all subgroups (Table 2); however, 'unhealthy' dietary patterns did not significantly increase the risk of diabetes among female participants, studies conducted in Eastern countries, Japanese or Chinese participants and studies with fewer than 10 years of follow-up (Table 3).

No evidence of publication bias was revealed by the funnel plots (Fig. 5(a) and (b)) and asymmetry tests for both dietary patterns (Egger's test: $P=0.96$ and $P=0.66$ for 'unhealthy' and 'healthy' dietary patterns, respectively; Begg's test: $P=0.89$ for 'unhealthy' dietary patterns and $P=0.807$ for 'healthy' dietary patterns). Sensitivity analysis showed that excluding none of the studies notably changed the summary effects or turned the summary effects to non-significant. 


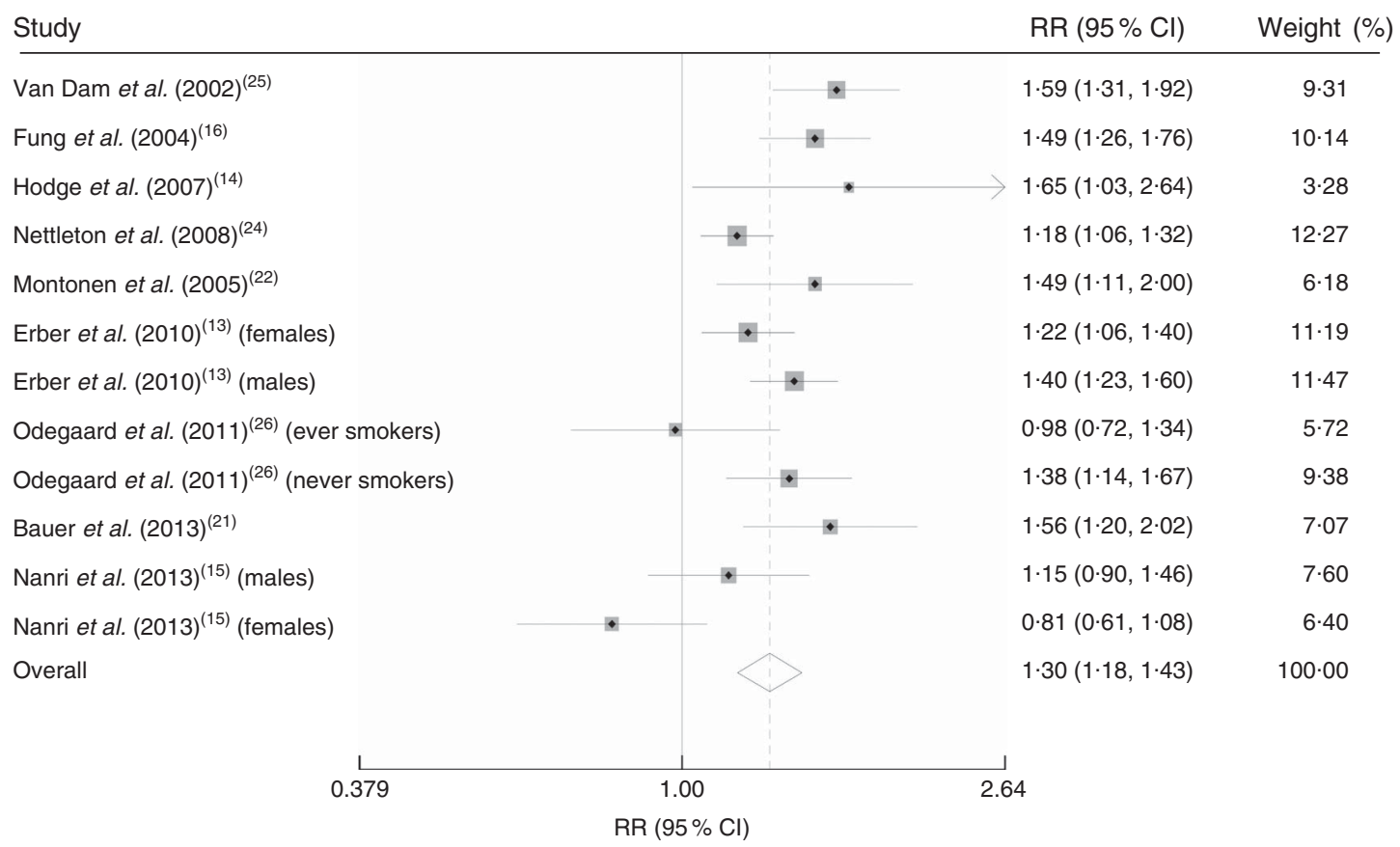

Fig. 3 Forest plot illustrating weighted relative risk (RR) using a random-effects model for the comparison of incident type 2 diabetes mellitus in participants with highest $v$. lowest adherence to 'unhealthy' dietary patterns. The study-specific RR and $95 \% \mathrm{Cl}$ are represented by the grey square and horizontal line, respectively; the area of the grey square is proportional to the specific-study weight to the overall meta-analysis. The centre of the diamond presents the pooled RR risk and its width represents the pooled $95 \% \mathrm{Cl}$

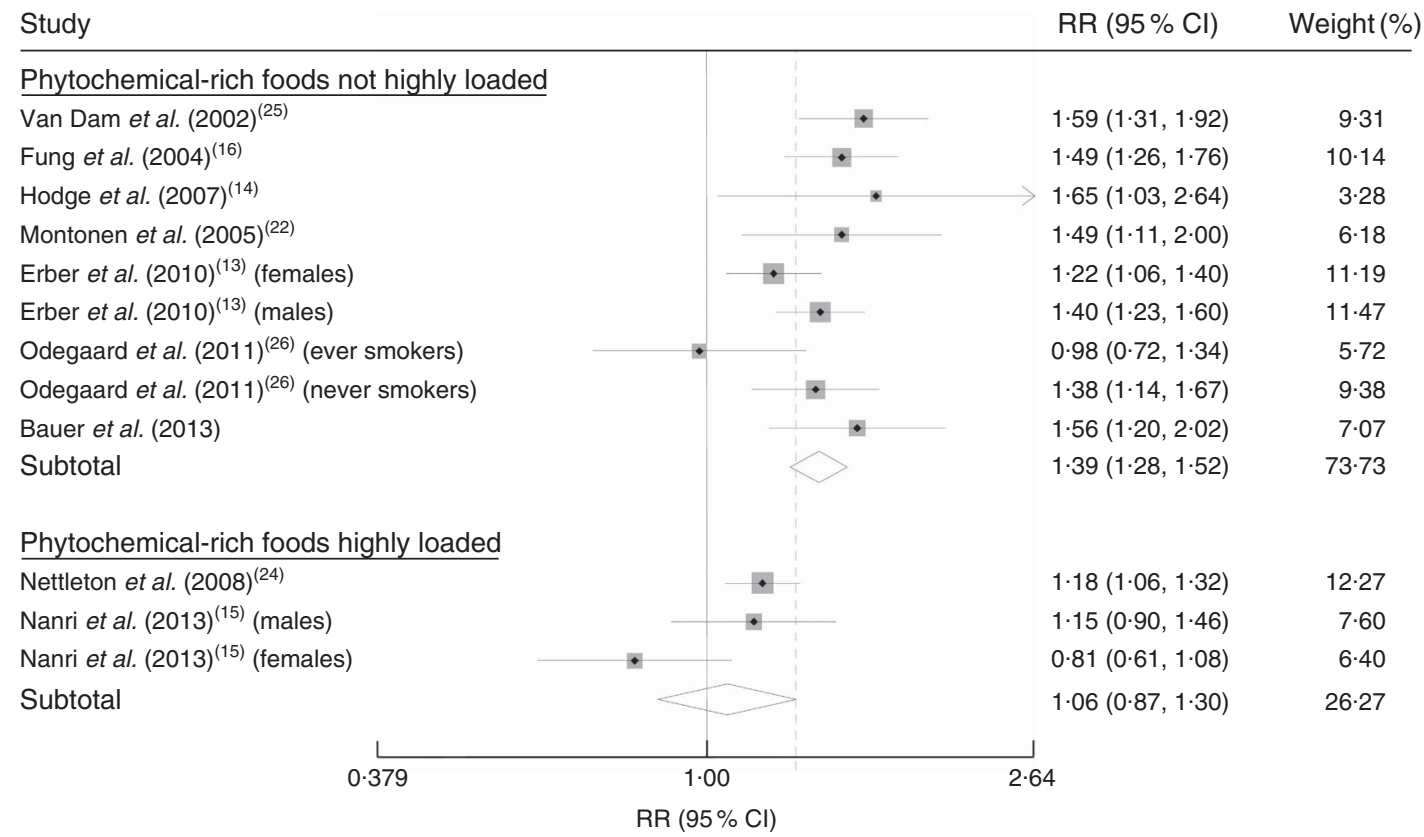

Fig. 4 Forest plot illustrating weighted relative risk (RR) using random-effects models for the comparison of incident type 2 diabetes mellitus in participants with highest $v$. lowest adherence to 'unhealthy' dietary patterns: subgroup analysis based on loading of foods with high phytochemical content in the 'unhealthy' dietary pattern. The study-specific RR and $95 \% \mathrm{Cl}$ are represented by the grey square and horizontal line, respectively; the area of the grey square is proportional to the specific-study weight to the overall metaanalysis. The centre of the diamond presents the pooled RR risk and its width represents the pooled $95 \% \mathrm{Cl}$

\section{Discussion}

Our meta-analysis of prospective cohort studies investigating the effects of dietary patterns on T2DM revealed that high adherence to a 'healthy' dietary pattern decreases diabetes risk by $14 \%$, while the risk of diabetes in individuals with high adherence to an 'unhealthy' dietary pattern is $30 \%$ greater than in individuals who have the lowest adherence. 


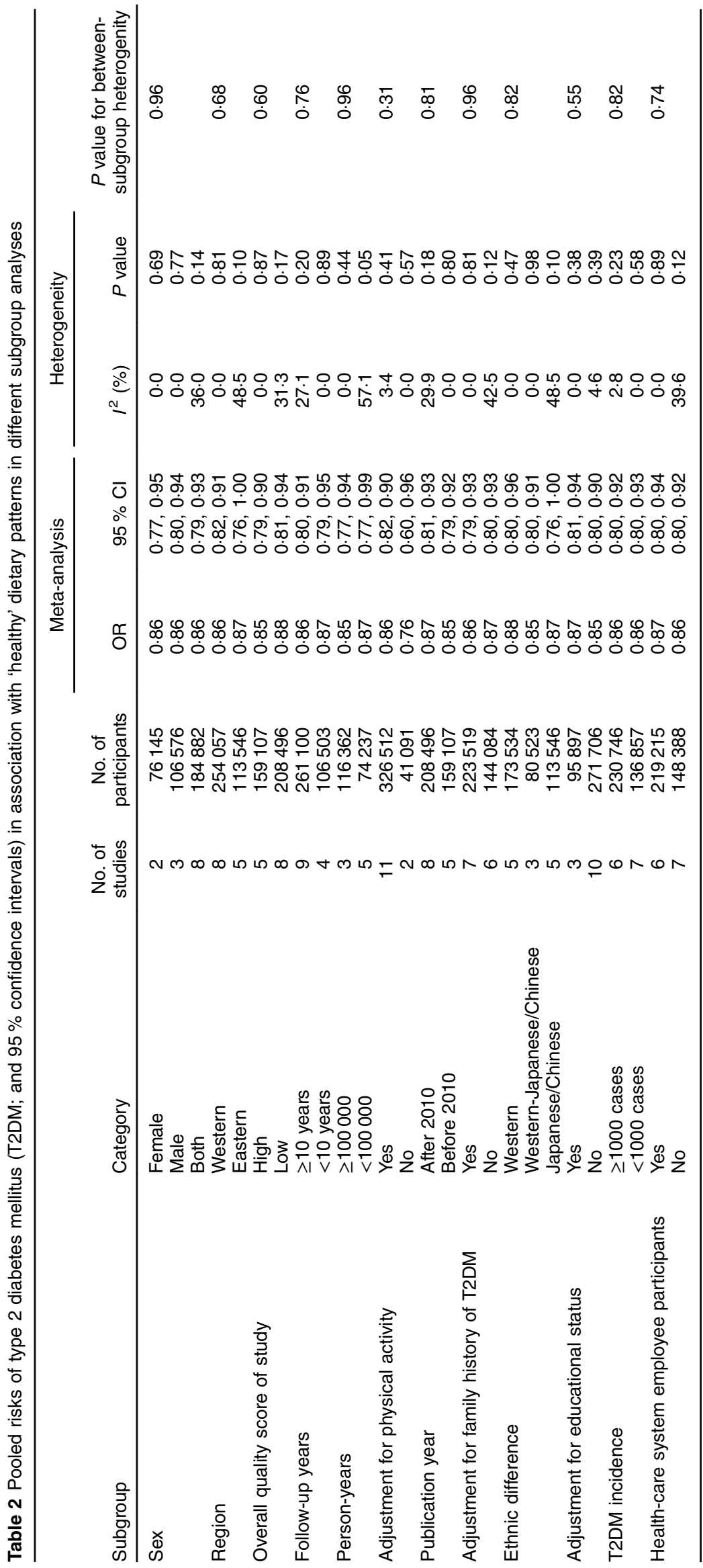




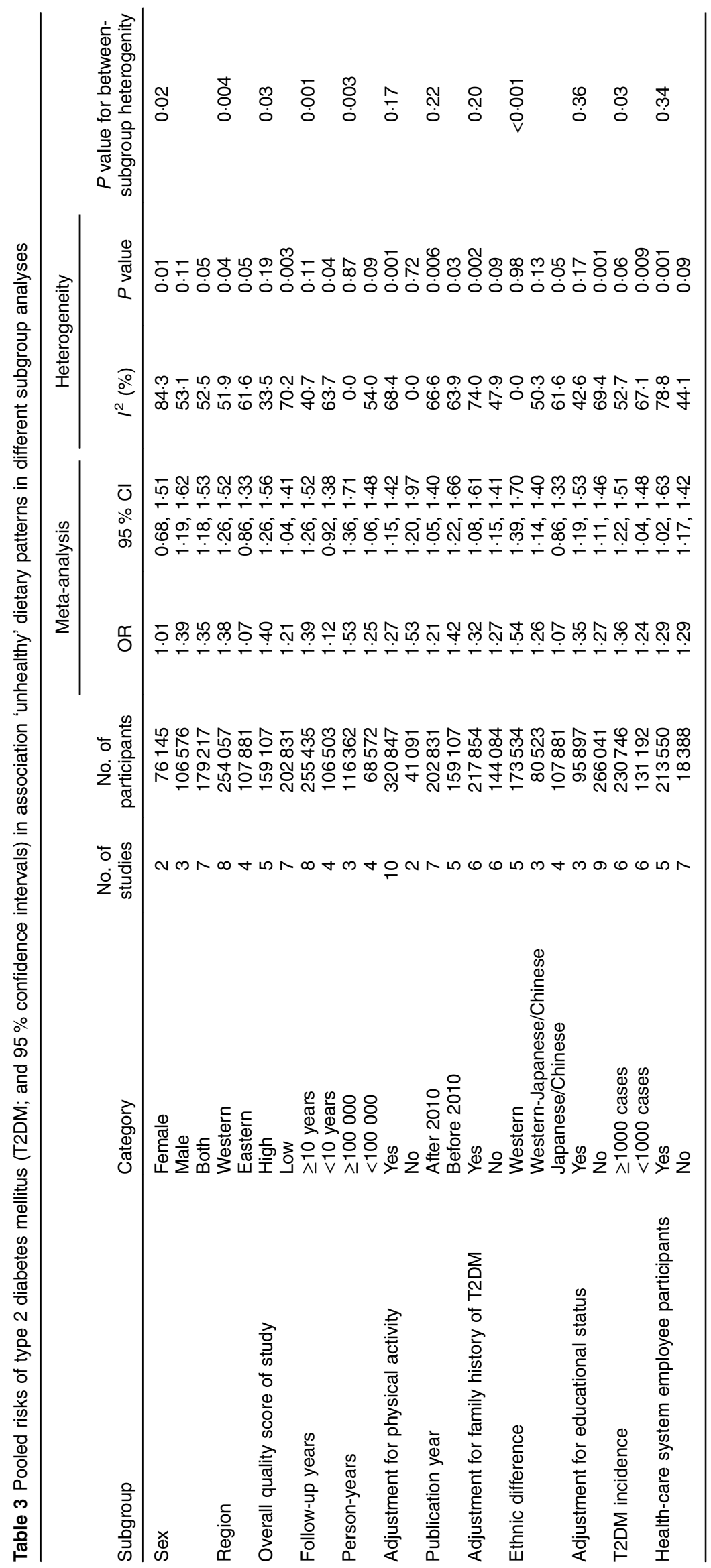



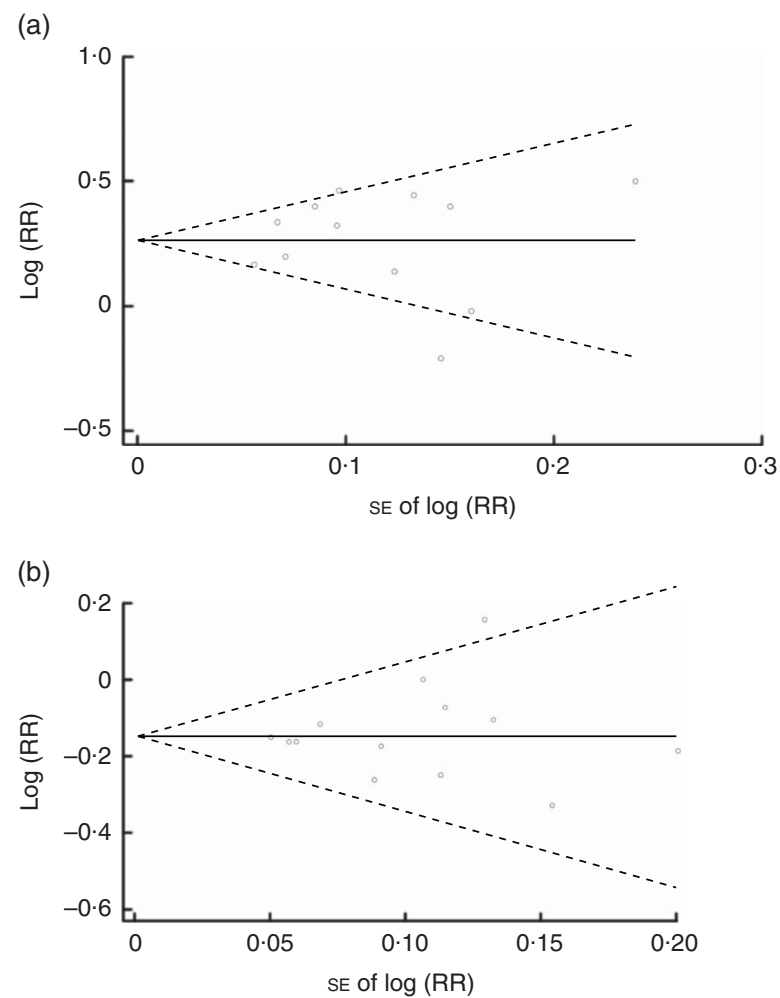

Fig. 5 Begg's funnel plot (with 95\% confidence intervals; - - -) of the logarithm of relative risk (RR) $v$. standard error of the RR in studies that assessed the effect of adherence to 'unhealthy' (a) and 'healthy' (b) dietary patterns on type 2 diabetes mellitus incidence. The horizontal line (-) shows the combined RR calculated with the random-effects model

'Healthy' dietary patterns, which are defined by higher consumption of vegetables, fruits, whole grains, low-fat dairy, fish, poultry and legumes, may play a protective role in T2DM incidence and these effects can be explained via their main nutrients such as antioxidants ${ }^{(27)}$, magnesium, potassium $^{(28)}$, carotenoids $^{(29)}$ and fibre content ${ }^{(28)}$. Also, it seems that several useful components such as flavones, isoflavones $^{(30)}$, lignans ${ }^{(31)}$ and phytates ${ }^{(32)}$ may have synergistic effects in protecting against diabetes incidence ${ }^{(33)}$.

'Unhealthy' dietary patterns are defined by high loadings of red meat, fried and processed foods ${ }^{(26,34)}$, French fries ${ }^{(35)}$ and sausage, as the major sources of saturated and trans-fatty acid contents ${ }^{(36)}$, and of foods with high glycaemic index $^{(37)}$ like refined grains ${ }^{(38)}$, sweets and desserts whose association with the risk of T2DM has been shown previously. Nitrate is one of the common added preservatives in ready-to-use meat products that can convert to nitrosamine and increase T2DM risk. Moreover, advanced glycation of high-fat products and meats can enhance oxidative stress and inflammatory factors that may be accompanied with insulin resistance and increase the possibility of developing $\mathrm{T}_{2} \mathrm{DM}^{(34)}$.

Our analysis showed that consumption of foods with high content of phytochemicals along with 'unhealthy' foods may change the effect of 'unhealthy' food patterns to non-significant. Phytochemicals like flavonoids, polyphenols, phenolic acids, phytates and lignans ${ }^{(39)}$ act as effective antioxidants and metal chelators ${ }^{(28)}$; in addition, the vitamins and minerals like magnesium ${ }^{(27)}$ and potassium $^{(28,40)}$ in these foods might counteract the harmful effects of other unhealthy items loaded on these patterns, which might explain this result.

Subgroup analysis showed significant differences according to sex. Risk of T2DM incidence in men with the highest adherence to 'unhealthy' dietary patterns was 39\% higher than in those with the lowest adherence, while this association was not significant in women. The oestrogen concentration of women might influence the overall effect $^{(41)}$. In the current analysis, long-term studies showed stronger health effects of the 'unhealthy' dietary pattern than studies which were done over a shorter period of $<10$ years. The effect of the 'unhealthy' dietary pattern in studies that followed-up for $\geq 10$ years showed a $39 \%$ higher risk of T2DM in participants with higher adherence to the 'unhealthy' dietary pattern compared with participants with lower adherence.

In Western countries including American, European and Australian adults, participants with the highest adherence to an 'unhealthy' dietary pattern, $v$. those with the lowest adherence, showed significantly different risk for T2DM as compared with their counterparts from Eastern countries. This observation was confirmed in the second subgroup analysis, which was performed based on ethnicity.

Based on our knowledge, there is one previous systematic review and meta-analysis that assessed the relationship between 'healthy' dietary patterns and diabetes risk in both a priori and a posteriori methods. In fact, these methods are very different. In the a priori method, researchers score participants' dietary intake based on defined food patterns, while the a posteriori analytical method tries to use statistical methods to derive dietary patterns from all consumed food items ${ }^{(41)}$. In comparison with our study, Alzahmi et al. included studies with different statistical analyses such as reduced rank regression, cluster analysis and factor analysis, and also studies that assessed the effects of different dietary indices ( $a$ priori dietary patterns), in relation to $\mathrm{T} 2 \mathrm{DM}$ risk $^{(42)}$. Including studies with different methods of assessing the dietary intake leads to a high degree of heterogeneity and a biased overall estimation of the effect of dietary patterns on T2DM. Furthermore, we tried to conduct several subgroup analyses to investigate possible sources of heterogeneity between studies included in our meta-analysis.

There are several limitations regarding the present review. Our findings might be affected by strengths and weaknesses of the study designs and measurement errors of the assessment tools. Measurement errors and misclassification arising from the various loading factors and variation in the extracted dietary patterns' type and number can be identified, too. Although there is the possibility of wide variation in conducting factor analysis, studies reflect a 
strong association between information derived from FFQ and diet histories. Reproducibility and validity of the used FFQ confirm the stability of the observed relationships. We selected patterns across different studies based on authors' identified name and similarity in food loadings, which helped us to minimize the risk of bias. In addition, there is some inconsistency in the confounding factors adjusted for in the association between dietary patterns and T2DM among included studies.

\section{Conclusion}

Our systematic review and meta-analysis provides confident evidence that following an 'unhealthy' dietary pattern including refined grains, processed and red meat, French fries, sweet and desserts can significantly increase T2DM incidence risk, while consuming higher amounts of vegetables, fruits, whole grains and seeds in the context of a 'healthy' dietary pattern can significantly reduce the risk of T2DM.

\section{Acknowledgements}

Financial support: This research received no specific grant from any funding agency in the public, commercial or notfor-profit sectors. Conflict of interest: None. Authorship: Z.A. and R.G. contributed in conception, design and manuscript drafting. Z.A. and A.S.-A. contributed in statistical analyses and data interpretation. All authors contributed in the approval of the final manuscript for submission. Ethics of human subject participation: Ethical approval was not required.

\section{References}

1. Zimmet P, Alberti KG \& Shaw J (2001) Global and societal implications of the diabetes epidemic. Nature $\mathbf{4 1 4}$, 782-787.

2. Yach D, Stuckler D \& Brownell KD (2006) Epidemiologic and economic consequences of the global epidemics of obesity and diabetes. Nat Med 12, 62-66.

3. King H, Aubert RE \& Herman WH (1998) Global burden of diabetes, 1995-2025: prevalence, numerical estimates, and projections. Diabetes Care 21, 1414-1431.

4. Harris MI, Flegal KM, Cowie CC et al. (1998) Prevalence of diabetes, impaired fasting glucose, and impaired glucose tolerance in US adults. The Third National Health and Nutrition Examination Survey, 1988-1994. Diabetes Care 21, 518-524.

5. Seidell JC (2000) Obesity, insulin resistance and diabetes - a worldwide epidemic. Br J Nutr 83, Suppl. 1, S5-S8.

6. World Health Organization (2013) Diabetes. Fact sheet no. 312. http://www.who.int/mediacentre/factsheets/fs312/en/ index.html (accessed October 2013).

7. Cooper AJ, Sharp SJ, Lentjes MA et al. (2012) A prospective study of the association between quantity and variety of fruit and vegetable intake and incident type 2 diabetes. Diabetes Care 35, 1293-1300.
8. Sluijs I, Beulens JW, van der AD et al. (2010) Dietary intake of total, animal, and vegetable protein and risk of type 2 diabetes in the European Prospective Investigation into Cancer and Nutrition (EPIC)-NL study. Diabetes Care $\mathbf{3 3}$, 43-48.

9. Villegas R, Shu XO, Gao YT et al. (2008) Vegetable but not fruit consumption reduces the risk of type 2 diabetes in Chinese women. J Nutr 138, 574-580.

10. Jacobs DR Jr \& Steffen LM (2003) Nutrients, foods, and dietary patterns as exposures in research: a framework for food synergy. Am J Clin Nutr 78, 3 Suppl., 508S-513S.

11. Newby PK \& Tucker KL (2004) Empirically derived eating patterns using factor or cluster analysis: a review. Nutr Rev 62, $177-203$.

12. Hu FB (2002) Dietary pattern analysis: a new direction in nutritional epidemiology. Curr Opin Lipidol 13, 3-9.

13. Erber E, Hopping BN, Grandinetti A et al. (2010) Dietary patterns and risk for diabetes: the multiethnic cohort. Diabetes Care 33, 532-538.

14. Hodge AM, English DR, O'Dea K et al. (2007) Dietary patterns and diabetes incidence in the Melbourne Collaborative Cohort Study. Am J Epidemiol 165, 603-610.

15. Nanri A, Shimazu T, Takachi R et al. (2013) Dietary patterns and type 2 diabetes in Japanese men and women: the Japan Public Health Center-based Prospective Study. Eur J Clin Nutr 67, 18-24.

16. Fung TT, Schulze M, Manson JE et al. (2004) Dietary patterns, meat intake, and the risk of type 2 diabetes in women. Arch Intern Med 164, 2235-2240.

17. Wells G, Shea B \& O'Connell D (2010) The NewcastleOttawa Scale (NOS) for Assessing the Quality of Nonrandomised Studies in Meta-Analyses. Ottawa, ON: Ottawa Health Research Institute.

18. Egger M, Smith GD \& Altman D (editors) (2008) Systematic Reviews in Health Care: Meta-Analysis in Context, 2nd ed. London: BMJ Books.

19. Higgins JP \& Thompson SG (2002) Quantifying heterogeneity in a meta-analysis. Stat Med 21, 1539-1558.

20. Egger M, Davey Smith G, Schneider M et al. (1997) Bias in meta-analysis detected by a simple, graphical test. BMJ $\mathbf{3 1 5}$, 629-634.

21. Bauer F, Beulens JW, van der A DL et al. (2013) Dietary patterns and the risk of type 2 diabetes in overweight and obese individuals. Eur J Nutr 52, 1127-1134.

22. Montonen J, Knekt P, Harkanen T et al. (2005) Dietary patterns and the incidence of type 2 diabetes. Am J Epidemiol 161, 219-227.

23. Morimoto A, Ohno Y, Tatsumi Y et al. (2012) Effects of healthy dietary pattern and other lifestyle factors on incidence of diabetes in a rural Japanese population. Asia Pac J Clin Nutr 21, 601-608.

24. Nettleton JA, Steffen LM, Ni H et al. (2008) Dietary patterns and risk of incident type 2 diabetes in the Multi-Ethnic Study of Atherosclerosis (MESA). Diabetes Care 31, 1777-1782.

25. van Dam RM, Rimm EB, Willett WC et al. (2002) Dietary patterns and risk for type 2 diabetes mellitus in US men. Ann Intern Med 136, 201-209.

26. Odegaard AO, Koh W-P, Butler LM et al. (2011) Dietary patterns and incident type 2 diabetes in Chinese men and women: the Singapore Chinese Health Study. Diabetes Care 34, 880-885.

27. Zhang Y, Lee ET, Cowan LD et al. (2011) Coffee consumption and the incidence of type 2 diabetes in men and women with normal glucose tolerance: the Strong Heart Study. Nutr Metab Cardiovasc Dis 21, 418-423.

28. van Dam RM (2006) Coffee and type 2 diabetes: from beans to beta-cells. Nutr Metab Cardiovasc Dis 16, 69-77.

29. Smith B, Wingard DL, Smith TC et al. (2006) Does coffee consumption reduce the risk of type 2 diabetes in individuals with impaired glucose? Diabetes Care 29, 2385-2390. 
30. Thompson S (2011) Effect of pinto, black and dark red kidney bean consumption as part of a meal on postprandial glucose in adults with type 2 diabetes. PhD Thesis, Arizona State University.

31. Kuhad A, Sharma S \& Chopra K (2008) Lycopene attenuates thermal hyperalgesia in a diabetic mouse model of neuropathic pain. Eur J Pain 12, 624-632.

32. Kuhad A, Sethi R \& Chopra K (2008) Lycopene attenuates diabetes-associated cognitive decline in rats. Life Sci $\mathbf{8 3}$, 128-134.

33. Villegas R, Yang G, Gao YT et al. (2010) Dietary patterns are associated with lower incidence of type 2 diabetes in middle-aged women: the Shanghai Women's Health Study. Int J Epidemiol 39, 889-899.

34. InterAct Consortium, Bendinelli B, Palli D et al. (2013) Association between dietary meat consumption and incident type 2 diabetes: the EPIC-InterAct study. Diabetologia 56, $47-59$.

35. Halton TL, Willett WC, Liu S et al. (2006) Potato and French fry consumption and risk of type 2 diabetes in women. $A m J$ Clin Nutr 83, 284-290.
36. Meyer KA, Kushi LH, Jacobs DR Jr et al. (2001) Dietary fat and incidence of type 2 diabetes in older Iowa women. Diabetes Care 24, 1528-1535.

37. Sahyoun NR, Anderson AL, Tylavsky FA et al. (2008) Dietary glycemic index and glycemic load and the risk of type 2 diabetes in older adults. Am J Clin Nutr 87, 126-131.

38. Sun Q, Spiegelman D, van Dam RM et al. (2010) White rice, brown rice, and risk of type 2 diabetes in US men and women. Arch Intern Med 170, 961-969.

39. Thompson SV, Winham DM \& Hutchins AM (2012) Bean and rice meals reduce postprandial glycemic response in adults with type 2 diabetes: a cross-over study. Nutr J 11, 23.

40. Winham D, Webb D \& Barr A (2008) Beans and good health. Nutr Today 43, 201-209.

41. Newby PK, Muller D, Hallfrisch J et al. (2003) Dietary patterns and changes in body mass index and waist circumference in adults. Am J Clin Nutr 77, 1417-1425.

42. Alhazmi A, Stojanovski E, McEvoy M et al. (2014) The association between dietary patterns and type 2 diabetes: a systematic review and meta-analysis of cohort studies. J Hum Nutr Diet 27, 251-260. 